\title{
Corrigendum: Plant cholesterol biosynthetic pathway overlaps with phytosterol metabolism
}

Prashant D. Sonawane, Jacob Pollier, Sayantan Panda, Jedrzej Szymanski, Hassan Massalha, Meital Yona, Tamar Unger, Sergey Malitsky, Philipp Arendt, Laurens Pauwels, Efrat Almekias-Siegl, Ilana Rogachev, Sagit Meir, Pablo D. Cárdenas, Athar Masri, Marina Petrikov, Hubert Schaller, Arthur A. Schaffer, Avinash Kamble, Ashok P. Giri, Alain Goossens and Asaph Aharoni

Nature Plants 3, 16205 (2016); published 22 December 2016; corrected 12 June 2017.

Two of the Supplementary Information files originally published contained errors. In Supplementary Table 1a, the SolycIDs for SMO1 and SMO2 were interchanged. In Supplementary Data 1, the SolycIDs for SMO3 and SMO4 were interchanged. Both errors have now been corrected. 